Normita S. Pangan, MD

Department of Otorhinolaryngology Head and Neck Surgery

East Avenue Medical Center
Correspondence: Dr. Normita S. Pangan Department of Otorhinolaryngology- Head and Neck Surgery 6th Floor, East Avenue Medical Center East Avenue, Diliman, Quezon City 1100 Philippines

Phone: (632) 9280611 loc 324

Fax: (632) 4356988

Email: normitap@yahoo.com

Reprints will not be available from the author.

The author declared that this represents original material that is not being considered for publication or has not been published or accepted for publication elsewhere, in full or in part, in print or electronic media; that the manuscript has been read and approved by the author, that the requirements for authorship have been met by the author, and that the author believes that the manuscript represents honest work.

Disclosures: The author signed a disclosure that there are no financial or other (including personal) relationships, intellectual passion, political or religious beliefs, and institutional affiliations that might lead to a conflict of interest.

\section{Arterio-Venous Malformation in the External Ear in Pregnancy}

Arterio-Venous Malformations (AVMs) are congenital vascular defects ${ }^{1}$ and are most commonly located in the head and neck area (81\%). ${ }^{2}$ The majority of these were localized over the cheek $(31 \%)$, ear( $16 \%)$, nose $(10 \%)$, forehead $(10 \%)$, upper lip (7\%), mandible $(5 \%)$, neck (5\%), and scalp and maxilla (4\%). ${ }^{2}$ There is equal predominance in males and females and no racial predilection. ${ }^{2}$ In general, there is "sparse literature about AVM in the external ear," let alone its occurrence in a pregnant patient.

Arterio-venous malformations are composed of a central nidus with anomalous arteriovenous shunts and a network of surrounding collateral vessels. ${ }^{4}$ The short circuit or shunting between the high pressure arterial and low pressure venous system accounts for much of the clinical presentation, anatomical changes and progression of the lesions. They are usually present at birth but commonly manifest in childhood or adolescence with gradual onset and progression and rarely can be associated with an enlarged heart and high output cardiac failure., ${ }^{3,5}$

The size of AVMs may increase rapidly secondary to infection, trauma, ligation or attempted excision and hormonal influences like pregnancy and puberty. ${ }^{3}$ We present the case of an AVM occurring in the external ear of a pregnant patient.

\section{CASE REPORT}

A 31-year-old G1P0 patient consulted with a uterine pregnancy of 24 weeks and two days age of gestation for an enlarging left external ear mass of approximately two years duration. The patient characterized the mass as initially "pimple-like", erythematous and nontender. There were no other associated signs and symptoms. The mass persisted despite taking Ciprofloxacin $500 \mathrm{mg}$ twice daily and Mefenamic Acid $500 \mathrm{mg}$ thrice daily for a week.

Four months prior to consult, on one of her pre-natal check-ups, the mass was now more visible with an increase in size to about $1.5 \times 1.5 \times 1.5 \mathrm{~cm}$. She noticed that her hearing was also decreased in the left ear. There were no other associated signs or symptoms. Against her obstetrician's advice to consult an otolaryngologist she decided to ignore the mass.

A month before consult, the mass was about $2 \times 2 \times 1.5 \mathrm{~cm}$, was more erythematous but nontender and had bled once (less than $3 \mathrm{~mL}$ ) after traumatic manipulation. The patient sought an ENT specialist and was advised to consult in our institution.

On examination the left external ear had a $2 \times 2 \times 1.5 \mathrm{~cm}$. erythematous, non-ulcerating mass of the external ear from tragus to external auditory canal. It was soft, compressible, nodular, nontender and pulsatile with a thrill and bruit. An AV malformation was considered. (Figure1)

Computed tomography axial and coronal sections revealed a soft tissue mass from tragus to external auditory canal with calcifications. (Figure 2) The rest of the ear structures were normal. Color-doppler ultrasonography showed a $2 \times 1.4 \times 1.8 \mathrm{~cm}$. congregation of small tubular structures in the subcutaneous area with the tubular mesh showing a diameter of 1.1 and $1.7 \mathrm{~mm}$. There was blood flow in the tubular structures with spectral tracing showing mixed arterial and venous flow 


\section{FEATURED GRAND ROUNDS}

Philippine Journal Of Otolaryngology-Head And Neck Surgery

Vol. 26 No. 2 July - December 2011

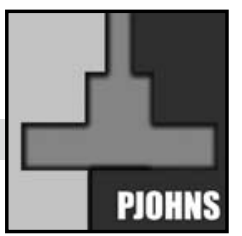

(arterio-venous malformation was considered). Magnetic resonance angiography (MRA) revealed a feeding vessel possibly from the external carotid artery. (Figure 3) Other examinations (2-D echocardiography and 12-lead ECG) were normal. Pure Tone Audiometry showed a moderately severe mixed hearing loss on the affected ear (left) and normal hearing on the contralateral side.

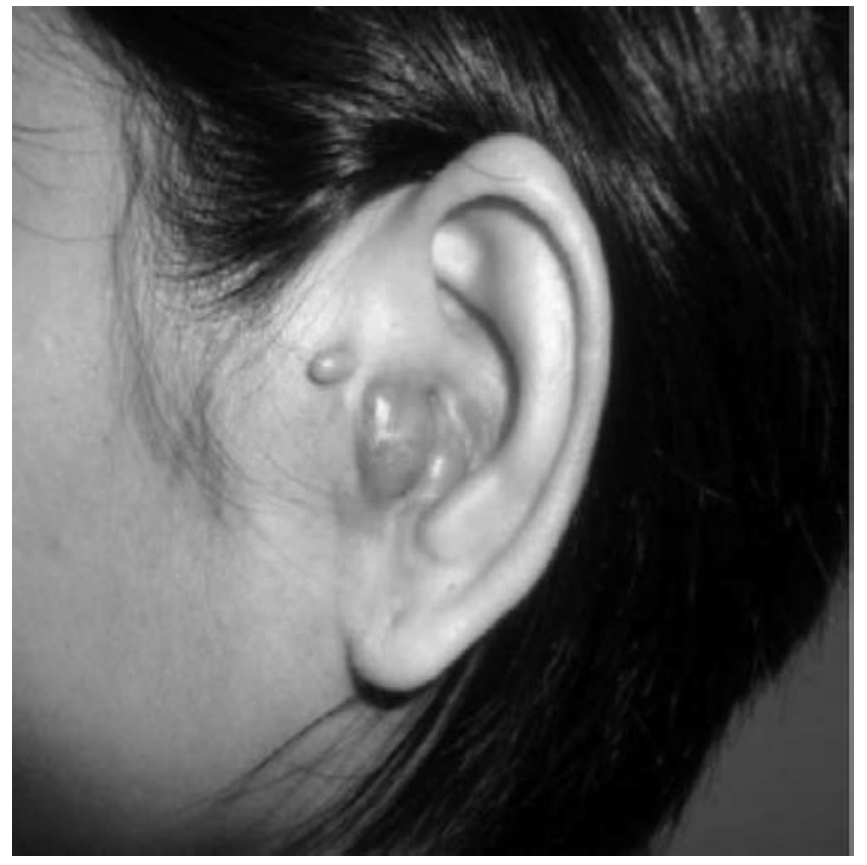

Figure 1. A $2 \times 2 \times 1.5 \mathrm{cms}$ erythematous, non-ulcerating mass of the external ear from tragus to external auditory canal. It was soft, compressible, nodular, nontender and pulsatile with a note of thril and bruit

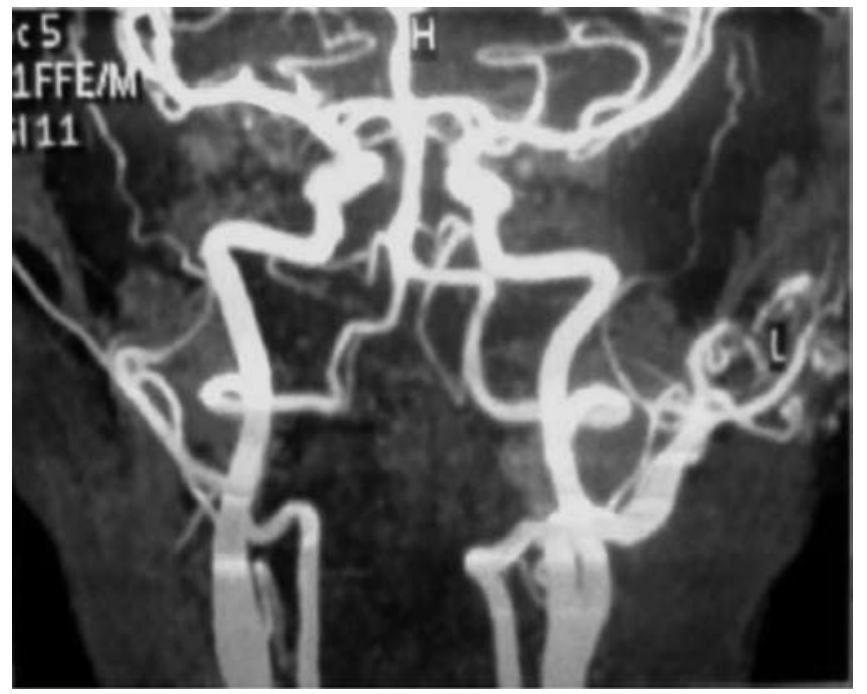

Figure 2-A. Magnetic resonance angiography of the left ear mass of the patient showing serpentine feeding vessels around the mass coming from external carotid artery.

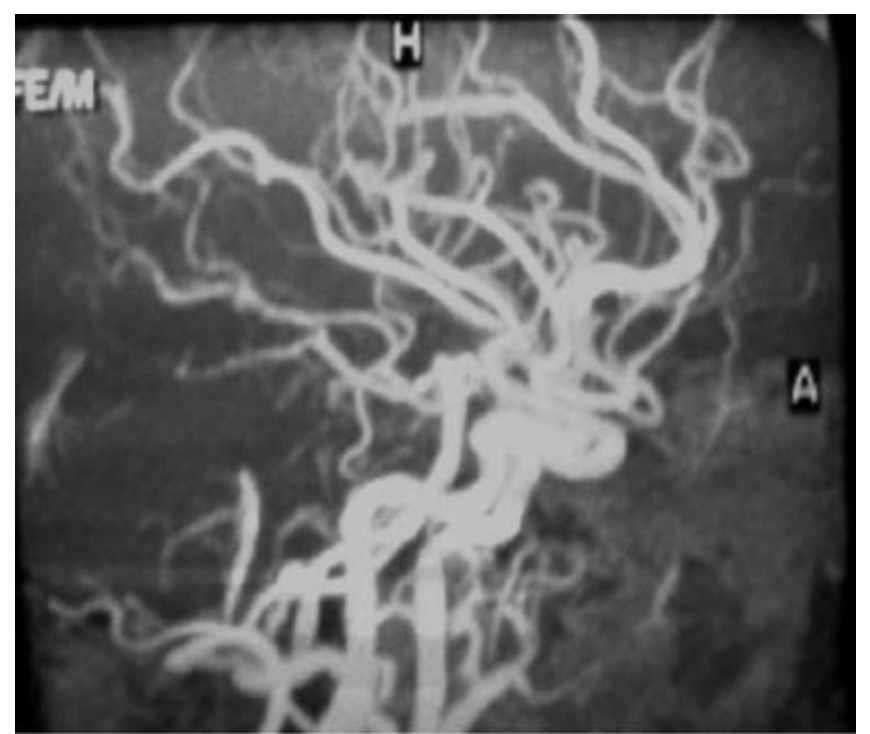

Figure 2-B. Magnified MRA of the left ear mass of the patient showing a central nidus of arterial and venous serpentine vessels.

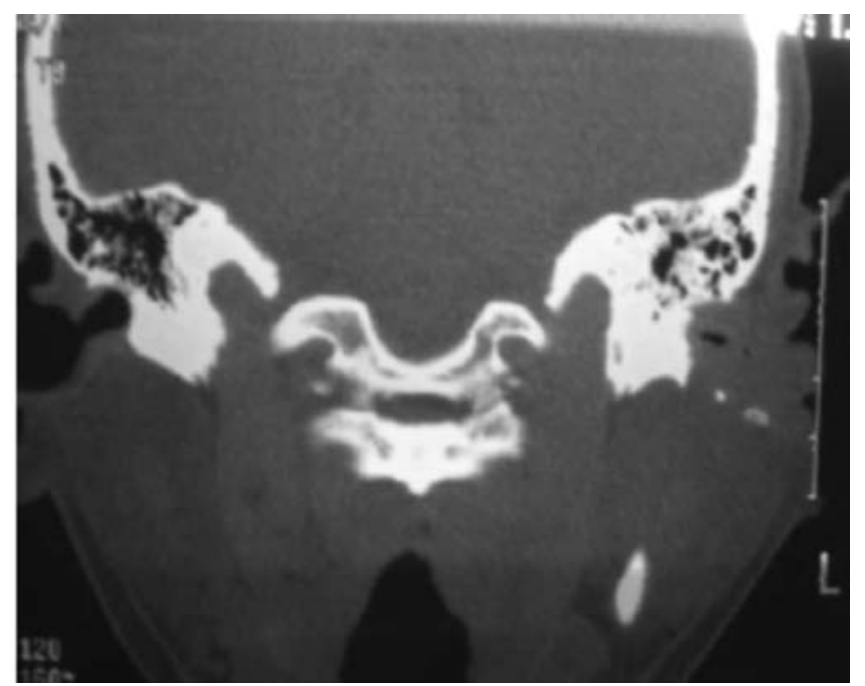

Figure 3. Temporal bone with contrast ct scan , coronal section (1 mm cut) showing left soft tissue mass from tragus to external auditory canal with noted calcifications

She was closely monitored through her 39th week of pregnancy when she delivered a healthy baby girl via caesarean section. No other complications or problems were encountered after and she was discharged on the eighth hospital day. We recommended excision of the AV malformation with reconstruction as a future treatment option.

\section{DICUSSION}

It has been estimated that the coexistence of pregnancy and AVM may result in a $150 \%$ increase in cardiac output above normal levels. ${ }^{5}$ Several cases of high output cardiac failure have been reported in pregnancies related to AVM and beginning a pregnancy with a 
pre-existing AVM can be dangerous. Progesterone increases venous distensibility during pregnancy and the menstrual cycle and may explain the relationship of AVM and pregnancy. The risk of AVM bleeding is 2 to $3 \%$ per year although we have found no record of bleeding risks in AVM of the external ear in pregnancy. Thus, our patient was closely monitored and was co-managed with her obstetrician on out-patient care. $^{3,4}$

Women with AVMs in pregnancy that were most likely to bleed tended to be younger (20-25 years) and were usually primiparous. Hemorrhages are most common between 15 and 20 weeks of gestation and could occur at any stage including during labour and in the puerperium. ${ }^{4}$ There is really no conclusive data regarding AVM hemorrhage risk during pregnancy. According to Ogilvy et al., the incidence of hemorrhage in pregnancy in patients with a prior history of hemorrhage is $5.8 \%$ during the year after the last menstrual period, but the number of patients with hemorrhage was small which made the data nondefinitive. Neither of these rates was significantly different from similar nonpregnant populations. ${ }^{4}$

Color doppler ultrasonography may be helpful in determining between high- and low- flow lesions in AVM. Magnetic resonance imaging (MRI) is said to be the diagnosis of choice since it depicts the extent and lack of invasion of these lesions providing multi-planar images and differentiating between high- and low-flow lesions. A Magnetic Resonance Angiogram (MRA) is the same non-invasive study but also examines the blood vessels as well as the structures of the brain, which is very advantageous as a guide if surgery is contemplated. ${ }^{5}$

Options for definitive management of AVM include observation, corticosteroid therapy, surgery and interferon (INF) therapy. Because the vast majority of AVM are small, well circumscribed and involute with no sequelae and acceptable cosmetic results, observation remains the preferred management option. Other treatments include surgery, endovascular therapy and radiosurgery. The latter can be used alone or in combination to treat an AVM. Superselective arterial or retrograde venous embolization may also be used as first choice treatment for AVM not amenable to surgery., ${ }^{3,5}$ Aside from conservative observation, none of these are advisable in pregnant patients. ${ }^{3,5}$

Hemorrhage during delivery has been a major concern of obstetricians and patients. However, the available data would suggest that in most cases, vaginal delivery does not carry a higher risk for hemorrhage than delivery by cesarean section. There are no data available to address whether cesarean section helps to reduce the already low incidence of AVM-associated complications during delivery, although there is evidence that increased venous pressure during a Valsalva maneuver is not directly transmitted to the draining veins. ${ }^{4} \mathrm{To}$ our knowledge, there is no existing management guideline for AVM in the external ear area in pregnancy. Treatment should be individually planned to minimize further AVM enlargement and cardiovascular complications, utilizing conservative means rather than surgery as a primary option until after the pregnancy has been carried to term.

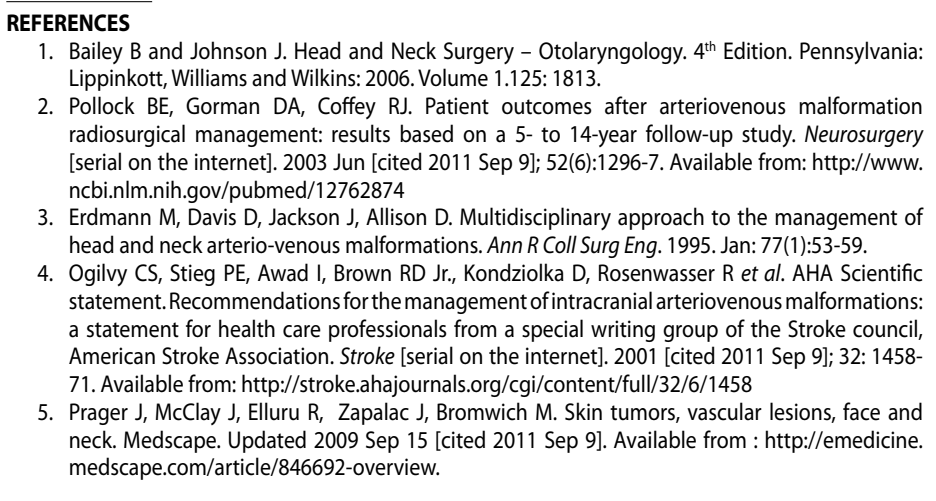

